\title{
Efficacy of Micronized Purified Flavonoid Fraction-Based Venoactive Therapy After Endovenous Mechanochemical Obliteration: Prospective Comparative Study
}

\author{
Vladimir Y. Khryshchanovich ${ }^{1}\left[\right.$ ] Yuri S. Nebylitsin $^{2}$ (1) $\cdot$ Vladimir A. Kosinets $^{2}$ (])
}

Accepted: 12 April 2021 / Published online: 28 April 2021

(C) The Author(s) 2021

\begin{abstract}
Background Endovenous interventions and minimally invasive open procedures are effective in the management of varicose veins, but can result in post-operative pain/discomfort.

Objective The objective of this study was to evaluate the clinical efficacy of micronized purified flavonoid fraction venoactive therapy for postoperative pain, vein-specific symptoms, and quality of life in patients with varicose veins following an endovenous mechanochemical ablation procedure.

Methods This prospective, observational, single-center study allocated patients into two groups: Group A, micronized purified flavonoid fraction $1000 \mathrm{mg}$ once daily for 30 days; Group B, no venoactive drug prescribed (control). The ClinicalEtiology-Anatomy-Pathophysiology classification system for chronic venous disorders was used to assess varicose veins; a 10-point Visual Analog Scale assessed pain syndrome intensity; the Venous Clinical Severity Score measured overall varicose vein severity; and the Chronic Venous Insufficiency QoL Questionnaire measured total quality of life.

Results The study enrolled 58 patients (mean age $36.9 \pm 4.1$ years; 24 men) with varicose veins of $\mathrm{C} 2-\mathrm{C} 4$ who underwent truncal mechanochemical ablation plus mini-phlebectomy or foam sclerotherapy. Group A had significantly lower pain syndrome at days 14 and 30 compared with Group B (1.76 vs 2.20, $p=0.039 ; 1.38$ vs 2.07, $p=0.003$, respectively), and clinical symptom severity at day 30 ( 2.67 vs $3.13, p=0.05$ ). Significant differences in quality-of-life scores existed between groups at days 14 and 30 ( 15.21 vs $18.75, p=0.008 ; 12.98$ vs $16.33, p=0.001)$. No micronized, purified flavonoid, fraction-related adverse effects were observed.

Conclusions Micronized purified flavonoid fraction-based venoactive adjuvant therapy after mechanochemical ablation alleviated pain, reduced the severity of symptoms, and improved the quality of life in patients with varicose veins.
\end{abstract}

\section{Introduction}

Endothermal techniques have proved to be effective for the treatment of incompetent truncal veins [1]. The tumescentless mechanochemical ablation (MOCA) technique has become an alternative treatment modality. Mechanochemical devices (ClariVein, Flebogrif) have been developed to minimize the negative aspects of both endothermal ablation and ultrasound-guided foam sclerotherapy for the treatment of saphenous incompetence, while incorporating

Vladimir Y. Khryshchanovich

vladimirkh77@mail.ru

1 Belarusian State Medical University, Dzerzhinski Ave., 83, Minsk 220116, Republic of Belarus

2 Vitebsk State Order of Peoples Friendship Medical University, Vitebsk, Republic of Belarus

\section{Key Points}

Venoactive adjuvant therapy with micronized purified flavonoid fraction at a standard daily dosage after an endovenous mechanochemical ablation procedure alleviates pain, reduces the severity of symptoms, and improves quality of life.

The benefits of venoactive adjuvant therapy include faster rehabilitation.

The better perception of the endovenous intervention could be attributable to the fact that mechanochemical ablation was performed with local anesthesia. 
the benefits of each [1-6]. Treatment with MOCA resulted in less postoperative pain/edema and a faster improvement in the Venous Clinical Severity Score (VCSS) in the short term but more hyperpigmentation compared with radiofrequency ablation (RFA) [7]. In addition, MOCA was associated with a significant reduction in rates of ecchymosis and hematoma formation and postoperative phlebitis and a shorter time to return to work compared with thermal ablation [2, 8]. In comparative studies, including the Mechanochemical endovenous Ablation to RADiOfrequeNcy Ablation (MARADONA) study [7], short-term (6-month) outcomes were similar with MOCA and RFA, but there were more anatomical failures between 6 months and 3 years after MOCA than after RFA [5-7]. However, compared with RFA, MOCA was associated with a lower incidence of nerve injury, deep vein thrombosis, and skin burns [5].

The most frequently reported complications in the MOCA studies were induration (12-18\%), thrombophlebitis $(2-13 \%)$, and ecchymosis $(8-10 \%)$ [1]. Less frequently reported were deep venous thrombosis $(0-1 \%)$ and hyperpigmentation (5\%). No other major complications have been reported using MOCA in the treatment of saphenous vein incompetence. Despite the low incidence of serious postoperative complications, many treatments for varicose veins (VVs) -including MOCA, modern open surgery, and endovenous thermal obliterationmay be associated with adverse effects such as pain, edema, hematoma, and burning. Venoactive treatments such as micronized purified flavonoid fraction (MPFF) have proven anti-edema, anti-inflammatory, and venoactive actions $[9,10]$ and are used from the early stages of VVs. These agents may also provide benefits in the postprocedural period after MOCA. Thus, in current guidelines on the management of chronic venous disorders in the lower limbs, MPFF carries a grade A recommendation for the relief of symptoms based on a high level of evidence [11].

Micronized purified flavonoid fraction is composed of five active flavonoids: micronized diosmin (accounting for $90 \%$ ), hesperidin, diosmetin, linarin, and isorhoifolin (the remaining 10\%) [12]. Their therapeutic effect is attained mainly through reducing capillary permeability, inhibiting the inflammatory cascade, and increasing venous tone. Micronized purified flavonoid fraction is associated with a reduction in edema and faster venous ulcer healing, while calcium dobesilate and rutosides are better at relieving leg cramps and edema, respectively [13]. A meta-analysis evaluating the effects of placebo and four venoactive drugs (MPFF, ruskus extract, hydroxyethylrutosides, diosmin) demonstrated the superior efficacy of MPFF on paramalleolar edema [14].
Evidence suggests that the anti-edema, anti-inflammatory, and venoactive actions of MPFF can help reduce pain and inflammatory syndromes in patients with chronic venous disease and after venous surgeries [9, 10, 15-17]. The European Society for Vascular Surgery guidelines on the management of patients with chronic venous diseases recommend MPFF and sulodexide as the main treatment (Class IIa, evidence level A) [10]. The International Union of Angiology guidelines also recommend MPFF combined with compression therapy for faster healing of venous ulcers in the lower limbs (Class 1, Level B) [18]. The pleiotropic pharmacologic effects of MPFF influence almost every component of VV pathogenesis-it suppresses endothelial cell activation and leukocyte adhesion, enhances capillary stability, inhibits free radicals and lysosomal enzymes, and normalizes prostaglandin E2 synthesis [15].

The use of venoactive drugs can facilitate the recovery process following saphenous vein high ligation and stripping, laser or RFA, and sclerotherapy [19]. Several openlabel clinical trials have demonstrated that venoactive drugs are effective after surgery, endovenous thermal ablation (laser, radiofrequency), and sclerotherapy [20-23]. The latest systematic review on the use of venoactive drugs in this setting illustrates the benefit of MPFF in terms of reducing post-procedural pain, hematoma count and area, and VV symptoms [19]. However, to the best of our knowledge, no studies have examined the effect of venoactive therapy on patient recovery following a non-thermal non-tumescent endovenous MOCA. This study was performed to evaluate the clinical efficacy of venoactive MPFF therapy for postoperative pain, symptoms, and quality of life (QoL) in patients with VV after endovenous MOCA.

\section{Materials and Methods}

\subsection{Participants and Study Design}

This was a single-center, observational, non-randomized study with a control group. Eligible patients were those who underwent surgery at the Vascular Surgery Department and received MPFF in Belarus for VV in the 12 months between 1 January and 31 December, 2019. The decision to take MPFF and enter the study was made by the patient based on information given by the physician. Enrolled patients were categorized on the basis of the (non-) use of adjuvant venoactive therapy in the postoperative period: Group A comprised those who received MPFF (Detralex ${ }^{\circledR}$; Servier, France) 1000 mg once daily for 30 days; Group B included patients who did not receive the venoactive drug.

The study inclusion criteria were as follows: age $>18$ years; Clinical-Etiology-Anatomy-Pathophysiology 
(CEAP) classification system for chronic venous disorders clinical class C2-C4 [24]; saphenofemoral/saphenopopliteal junction (SFJ/SPJ) insufficiency and pathological $(>0.5 \mathrm{~s})$ reflux in the great/small saphenous vein $(\mathrm{GSV} /$ SSV) manifested by one or more 'venous' symptoms (pain, itching, nocturnal leg cramps, swelling/pulsing sensation, heaviness, fatigue, and overall discomfort), vein diameter at the $\mathrm{SFJ} / \mathrm{SPJ}>4.5 \mathrm{~mm}$ and $<8 \mathrm{~mm}$ in an upright position.

To minimize the chance of selection bias, the exclusion criteria were: isolated reflux in tributaries, GSV/SSV tortuosity, known allergy to the sclerosant, deep/superficial vein thrombosis, post-thrombotic occlusion, confirmed thrombophilia, postoperative VV recurrence, obliterating peripheral artery diseases (ankle-brachial pressure index $<0.8$ ), pregnancy, and lactation. To avoid confounding caused by pharmacological interactions, patients were also excluded if they received concomitant treatment with any venoactive drug intake within 3 months prior to the start of the study and an allergy or hypersensitivity to MPFF.

The pre-operative examination documented patient history (including the information about prior venous surgeries), identifying clinical signs/symptoms of VV, duplex ultrasound evaluation of the lower extremities, and a clinical-etiological and anatomical-pathophysiological assessment based on the CEAP criteria. The primary transverse and longitudinal ultrasound examination (B-mode, color, and spectral Doppler) was performed by doctors at the Vascular Pathology Non-Invasive Diagnostics Unit before deciding on treatment choices. Reflux in the SFJ/SPJ area was determined in supine and upright positions using the Valsalva maneuver or manual compression/decompression test, respectively. The SFJ/SPJ and GSV/SSV segments with retrograde blood flow longer than $0.5 \mathrm{~s}$ were considered incompetent. Venous reflux section length and incompetent GSV/SSV segments' diameter (in $\mathrm{mm}$ ) were reflected in medical documentation.

The patients were informed about the MOCA technique, the intervention's potential complications, and adverse effects. Signed consent forms were obtained from all individual participants included in the study. The study was approved by the Belarusian State Medical University's Biomedical Ethics Committee (approval number 20140455).

\subsection{Interventions}

\subsubsection{Surgical Intervention}

Following the registration of the Flebogrif ${ }^{\mathrm{TM}}$ device in Belarus in 2015, it was widely used in conjunction with MPFF after MOCA. The Flebogrif ${ }^{\mathrm{TM}}$ catheter (Balton Sp. $\mathrm{z}$ o.o., Warsaw, Poland) is an endoluminal non-thermal nontumescent device designed to cause occlusion (fibrosis) of the target vein; this is achieved with retractable cutters and foamed sclerosant that result in mechanical and chemical damage to the endothelial lining of the vein.

The Flebogrif ${ }^{\mathrm{TM}}$ device's design is based on a $5 \mathrm{Fr}$ singlechannel diagnostic vascular catheter 60 or $90 \mathrm{~cm}$ long (with $1-\mathrm{cm}$ graduated marks), its lumen containing a retractable metal rod with five sharp wires ('claws') attached to its tip. As the 'claws' are released and the catheter is withdrawn from the vessel, the endothelial layer of the vein walls are damaged; at this time, the distance between the fully opened cutting elements is $\sim 29 \mathrm{~mm}$, which is sufficient to treat incompetent veins up to $17-20 \mathrm{~mm}$ in diameter [19].

A dose of 3\% lauromacrogol-400 foamed solution (Ethoxysclerol ${ }^{\circledR}$; Kreussler \& Co. GmbH, Wiesbaden, Germany) was prepared using the method described by Tessari and colleagues [25]. This method uses a three-way tap adapted to two $10-\mathrm{mL}$ syringes to $\mathrm{mix} 2 \mathrm{~mL}$ of liquid sclerosant with $8 \mathrm{~mL}$ of room air (in 1:4 proportion) to obtain a mixture of $10 \mathrm{~mL}$.

The patients were placed in supine or prone positions for GSV or SSV ablation, respectively. The catheter length choice was determined by that of the incompetent vein segment. The GSV/SSV was punctured with a straight needle $18 \mathrm{G}$ at the distal point of reflux in an operating room under local anesthesia (1\% lidocaine hydrochloride solution) and ultrasound guidance, after which a 0.035 " J-shape guidewire was employed to insert a $6 \mathrm{Fr}$ introducer with an expander. Upon the removal of the introducer, a Flebogrif ${ }^{\mathrm{TM}}$ catheter was delivered to the lumen of the target vein with the tip positioned 2-3 cm below the SFJ/SPJ. Following the guidewire's removal, the catheter's cutting elements were released, shifting its outer 'shell' against the stationary inner rod, after which the syringe containing the $10 \mathrm{~mL}$ of foam was connected to the central channel's port [26]. The catheter was being distally removed from the vein in a smooth motion all the way up to the introducer, damaging the endothelium with its cutters, with simultaneous introduction of the foam $(0.1-0.2 \mathrm{~mL}$ per $1 \mathrm{~cm})$. The graduated marks on the catheter helped measure the treated GSV/SSV section's length. During and within $5 \mathrm{~min}$ after the foam injection, the ultrasound probe was used to apply percutaneous compression in the SFJ/SPJ plane. Where deemed necessary, MOCA was supplemented with simultaneous Müller-Varadi mini-phlebectomy or standard foam sclerotherapy of the visible varicose tributaries. Immediately after the procedure, a class 2 RAL compression stocking was place on the operated limb, and the patients were recommended to take a 30-min walk. The use of elastic compression was continuous over the first $24 \mathrm{~h}$, with subsequent transition to daytime wear for 4 weeks.

Dynamic follow-up of both groups' patients (visual examination, limb photography, ultrasound examination) was performed at days 7,14 , and 30 , respectively, \pm 1 day 
after their surgery. Technical success was defined as the ability to successfully complete the procedure (i.e., the catheter safely placed at the defined location and the GSV/SSV treated without technical problems). Anatomical success was defined by complete occlusion of the treated GSV/SSV segment, objectively assessed by duplex ultrasound.

\subsubsection{MPFF Treatment}

Patients in Group A received MPFF 1000 mg once daily for 30 days, with the first dose taken on the day of surgery. The 1000-mg tablet was to be taken orally and with food at lunchtime.

\subsection{Outcome Assessments}

Before the procedure and at each visit, adverse effects and intervention-related complications, pain syndrome intensity [on a 10-point Visual Analog Scale (VAS)], the VCCS [27], and QoL scores were recorded. The total QoL index was determined with the Chronic Venous Insufficiency Questionnaire (CIVIQ-20) using the modified formula [ $(S-20) \times 1.25]$, where $S$ was the sum of points scored after answering each of the 20 questions [28]. Thus, one point indicated the absence of symptoms or subjective feelings, whereas five points corresponded to their maximum severity. The endpoints for this study were pain intensity, QoL, and VCSS at days 7, 14, and 30 after MOCA.

\subsection{Statistical Analysis}

The data on all VV patients were collated from the electronic database "AIS Clinic" and subjected to further analysis using the Statistica Version 10 and SPSS Version 23 software. The statistical significance was assessed using widely accepted nonparametric tests: the Mann-Whitney $U$ test for pairwise comparison of variables in independent samples, the sign rank test for pairwise comparison of variables in dependent samples, and the Kruskal-Wallis test to compare three or more variables in independent samples. The central tendency and the spread of quantitative indicators were indicated as arithmetic mean and 95\% confidence interval. The differences identified were considered statistically significant at $p \leq 0.05$.

Multivariate regression analysis was applied to the patient data from Groups A and B using results for three indices (pain severity, VCSS, and QoL). The following variables were included: sex, age, the number of legs treated, the vein diameter, the SSV treatment, and simultaneous sclerotherapy.

\section{Results}

\subsection{Patients' Clinical and Demographic Characteristics}

The study included 58 patients with CEAP class C2-C4, mean age was $36.9 \pm 4.1$ years, and $34(58.6 \%)$ were women; 43 were in Group A (treated with MPFF) and 15 in Group B (control); the approximate ratio between Group A and Group B was 3:1. A clinically significant GSV/SSV insufficiency in one or both lower extremities was diagnosed in all cases. Patient distribution by the CEAP clinical class $(\mathrm{C} 2: \mathrm{C} 3: \mathrm{C} 4)$ was 44:8:6 (Table 1). The most common symptoms patients were experiencing were pain (Group A: $74.4 \%$, Group B: 66.7\%), heaviness (Group A: 25.6\%, Group B: 26.7\%), and fatigue (Group A: 23.3\%, Group B: 26.7\%).

A total of 65 saphenous trunks were treated in 58 patients, while 48 patients $(82.7 \%)$ underwent surgery for unilateral GSV insufficiency; seven patients (12.1\%) and three patients (5.2\%) for bilateral lesion of GSV and SSV, respectively. Mini-phlebectomy or foam sclerotherapy of tributaries was performed in $51(87.9 \%)$ and $2(3.4 \%)$ cases, respectively (Table 1). There were no cases of device-associated complications: following the successful GSV/SSV puncture, the Flebogrif ${ }^{\mathrm{TM}}$ catheter was inserted into the venous lumen and positioned as required in all cases (100\% technical success). The patients were discharged on the day of their surgery or the following day. There were no MOCA-associated thrombotic, neurological, or infectious complications recorded throughout the follow-up period. One month post-surgery, complete closure of the lumen of the GSV/SSV was confirmed in $100 \%$ of cases.

\subsection{Treatment Outcomes}

The initial pain syndrome intensity was comparable in Groups A and B, showing no significant difference ( 2.95 vs $3.27, p=0.29$ ). At days 7,14 , and 30 after the intervention, a significant effect on pain relief was observed in both groups ( $p<0.01$ for all three time points; day 30 values were 1.38 in Group A and 2.07 in Group B); at day 14 and then day 30 , there was a significant difference between groups, in favor of Group A, for pain relief (Fig. 1). Two patients in Group A (4.6\%) and two patients in Group B (13.3\%) reported pain on day 30 . There were qualitative differences in pre-operative and post-operative pain perception. According to the patients, the pain was initially caused by heaviness and edema of the lower extremities, whereas after the procedure, the pain was perceived as a consequence of the surgery rather than residual venous symptoms.

Before intervention, the cumulative VCSS index was similar in Groups A and B (4.14 vs 4.33 points, $p=0.55$ ). 
Table 1 Patients' clinical and demographic characteristics

\begin{tabular}{|c|c|c|c|}
\hline Characteristics & $\begin{array}{l}\text { Group A } \\
N=43\end{array}$ & $\begin{array}{l}\text { Group B } \\
N=15\end{array}$ & $P$ value \\
\hline Age, years, mean $\pm S D$ & $36.4 \pm 12.4$ & $37.2 \pm 9.6$ & 0.96 \\
\hline Sex, male/female, $n / n$ & $18 / 25$ & $6 / 9$ & 1.0 \\
\hline $\mathrm{C} 2, n(\%)$ & $31(72.1)$ & $14(3.3)$ & 0.66 \\
\hline $\mathrm{C} 3, n(\%)$ & $7(16.3)$ & $1(6.7)$ & 0.67 \\
\hline $\mathrm{C} 4 \mathrm{a}, n(\%)$ & $3(7.0)$ & - & 0.40 \\
\hline $\mathrm{C} 4 \mathrm{~b}, n(\%)$ & $2(4.7)$ & $1(6.7)$ & 1.0 \\
\hline Severity of pain before MOCA, mean of VAS score & $2.95 \pm 0.38$ & $3.27 \pm 0.49$ & 0.29 \\
\hline \multicolumn{4}{|l|}{ Symptoms of chronic venous disease, $n(\%)$} \\
\hline Pain & $32(74.4)$ & $10(67)$ & 0.73 \\
\hline Swelling/pulsing sensation & $7(16.3)$ & $1(6.7)$ & 0.67 \\
\hline Heaviness & $11(25.6)$ & $4(26.7)$ & 1.0 \\
\hline Overall discomfort & $9(20.9)$ & $3(20.0)$ & 1.0 \\
\hline Fatigue & $10(23.3)$ & $4(26.7)$ & 1.0 \\
\hline Itching & $4(9.3)$ & $1(6.7)$ & 1.0 \\
\hline Nocturnal leg cramps & $5(11.6)$ & $3(20.0)$ & 0.41 \\
\hline Unilateral lesion, $n(\%)$ & $37(86.0)$ & $14(93.3)$ & 0.66 \\
\hline Bilateral lesion, $n(\%)$ & $6(14.0)$ & $1(6.7)$ & 0.66 \\
\hline GSV reflux, $n$ of limbs & 46 & 16 & 1.0 \\
\hline SSV reflux, $n$ of limbs & 3 & - & 0.29 \\
\hline Diameter of GSV and SSV in $\mathrm{mm}$, mean \pm SD & $6.3 \pm 0.91$ & $6.13 \pm 1.09$ & 0.47 \\
\hline Number of interventions & 49 & 16 & 0.65 \\
\hline \multicolumn{4}{|l|}{ Intervention type, $n(\%)$ of patients } \\
\hline MOCA alone & $3(7.0)$ & $2(13.3)$ & 0.60 \\
\hline MOCA + mini-phlebectomy & $38(88.4)$ & $13(86.6)$ & 1.0 \\
\hline MOCA + sclerotherapy of tributaries & $2(4.7)$ & - & 1.0 \\
\hline
\end{tabular}

$G S V$ great saphenous vein, $M O C A$ mechanochemical ablation, $S D$ standard deviation, $S S V$ small saphenous vein, $V A S$ Visual Analog Scale
A continuous decline in the VCSS index was observed postoperatively at each follow-up time point in Groups A and $\mathrm{B}(p<0.01$ for all three time points); however, statistically significant differences between the groups were recorded at day 30 ( 2.67 vs $3.13, p=0.05$; Fig. 2 ). Following endovenous obliteration, the symptoms associated with heaviness and swelling in the lower limbs were resolved in almost all patients during the 30-day follow-up period; at day 30, one patient each in Group A (2.3\%) and Group B (6.7\%) reported swelling/pulsing sensation, and one patient in Group A (2.3\%) and two in Group B (13.3\%) reported heaviness. Overall discomfort was reported by one patient in Group A (2.3\%) and two in Group B (13.3\%). None of the patients in either group reported itching on day 30 . Fatigue was reported by one patient and nocturnal leg cramps by two patients, all of whom were in Group B; none of the patients in Group B reported these two symptoms on day 30. The incidence of symptoms on day 30 did not differ significantly between Group A and Group B.

There was no difference between baseline QoL values between Group A and Group B (23.15 vs 24.67; $p=0.46$; Fig. 3). In both groups, post-operative QoL significantly improved at each subsequent follow-up time point $(p<0.001)$. At day 14 and day 30 after surgery, total QoL was significantly higher in Group A compared with Group B (15.21 vs $18.75, p<0.001 ; 12.98$ vs 16.33 , $p=0.001)$. No MPFF therapy-related adverse effects were observed.

\subsection{Multivariate Regression Analysis}

None of the assessed variables had a significant influence on the VAS score (pain severity) in either Group A or Group B (Table S1 of the Electronic Supplementary Material). However, sex significantly influenced the VCSS index in both groups, with women showing a significantly lower response than men.

Small saphenous vein ablation showed a weak but direct correlation with the VCSS index and QoL in Group B only. Sclerotherapy showed an even weaker, but indirect correlation with just one out of three indices (VCSS). 


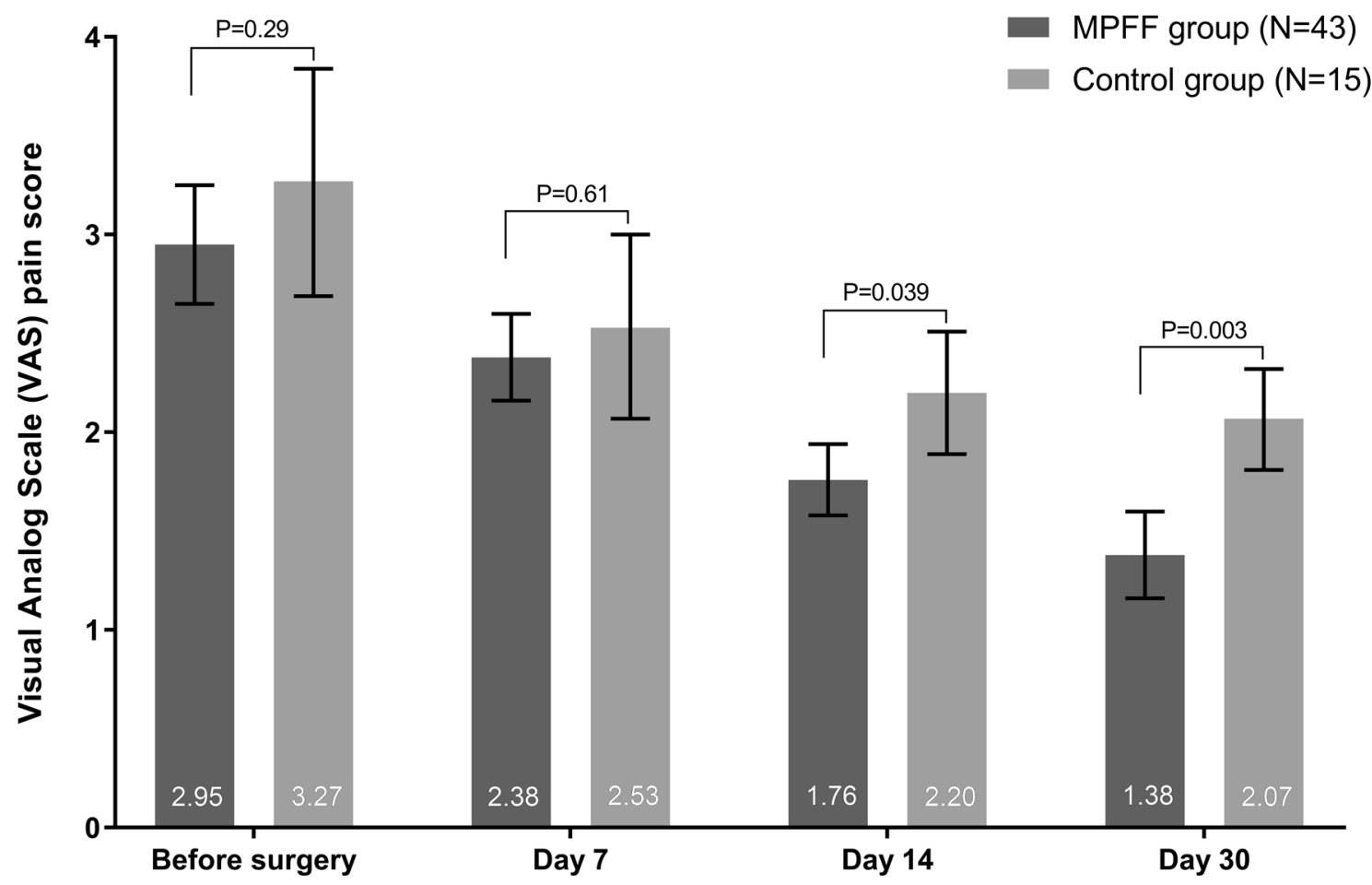

Fig. 1 Visual Analog Scale (VAS) pain scores: comparative cross-group analysis. Error bars represent $95 \%$ confidence intervals. $M P F F$ micronized purified flavonoid fraction

\section{Discussion}

In this observational single-center study in 58 patients with VV following an endovenous MOCA procedure, adjuvant treatment with MPFF for 4 weeks significantly reduced pain syndrome intensity and clinical symptom severity and improved QoL. The role of venoactive MPFF therapy after combined phlebectomy or endovascular interventions was studied in the DEFANCE and DECISION clinical trials $[20,22]$ and the results of our study are consistent with these trials. The non-randomized multicenter DEFANCE trial (Daflon ${ }^{\circledR} 500 \mathrm{mg}$, assEssment of eFficacy and safety for Combined phlEbectomy) confirmed the efficacy of perioperative ( 2 weeks before and 4 weeks after surgery) MPFF use after GSV stripping [20]. A significant reduction in the number and area of hematomas, relief of pain, and heaviness in the operated limb was recorded in the venoactive therapy group $(n=200)$ at days 7 and 30. At the same time, the results of the survey using the CIVIQ vein-specific questionnaire showed no significant QoL differences between the groups of MPFF and non-MPFF patients. The pain scores at 1 week post-MOCA in our study are higher than those reported in previous trials with MOCA alone [29], but are similar to those in studies of MOCA combined with sclerotherapy [20]. The authors attribute some inconsistency in the obtained data to the peculiarities of the postoperative period: the patient's well-being was more influenced by surgical trauma and the wearing of compression bandages than by the venous pathology manifestations. The efficacy of MPFF was evaluated in a randomized open-label DECISION trial based on the VCSS scale and the CIVIQ-14 questionnaire in 126 patients with VV CEAP clinical class C2-C4 after an endovascular intervention (laser or radiofrequency obliteration, US-guided foam sclerotherapy) [22]. The control group included 104 patients who underwent surgery and did not receive MPFF in the perioperative period. The VCSS and QoL indicators were better in the MPFF venoactive therapy group than in the control group at days 14 and 28 after the intervention. Our own observations are consistent with the main trends identified in the DEFANCE and DECISION trials [20, 22]. Certainly, the endovenous intervention per se helped alleviate the CVD symptoms and improve the patients' QoL. However, further benefits of a 30-day MPFF course were demonstrated in 43 patients with VV grade $\mathrm{C} 2-\mathrm{C} 4$, including significant pain reduction and QoL improvement at days 14 and 30, as well as marked relief of CVD symptoms 30 days after MOCA.

The results were, for the most part, not significantly influenced by patient demographic or clinical variables, as shown by the multivariate regression analysis, with the studied confounders showing weak and irregular effects on efficacy indices. However, we did find that women were significantly less likely than men to show a response on the VCSS. The 


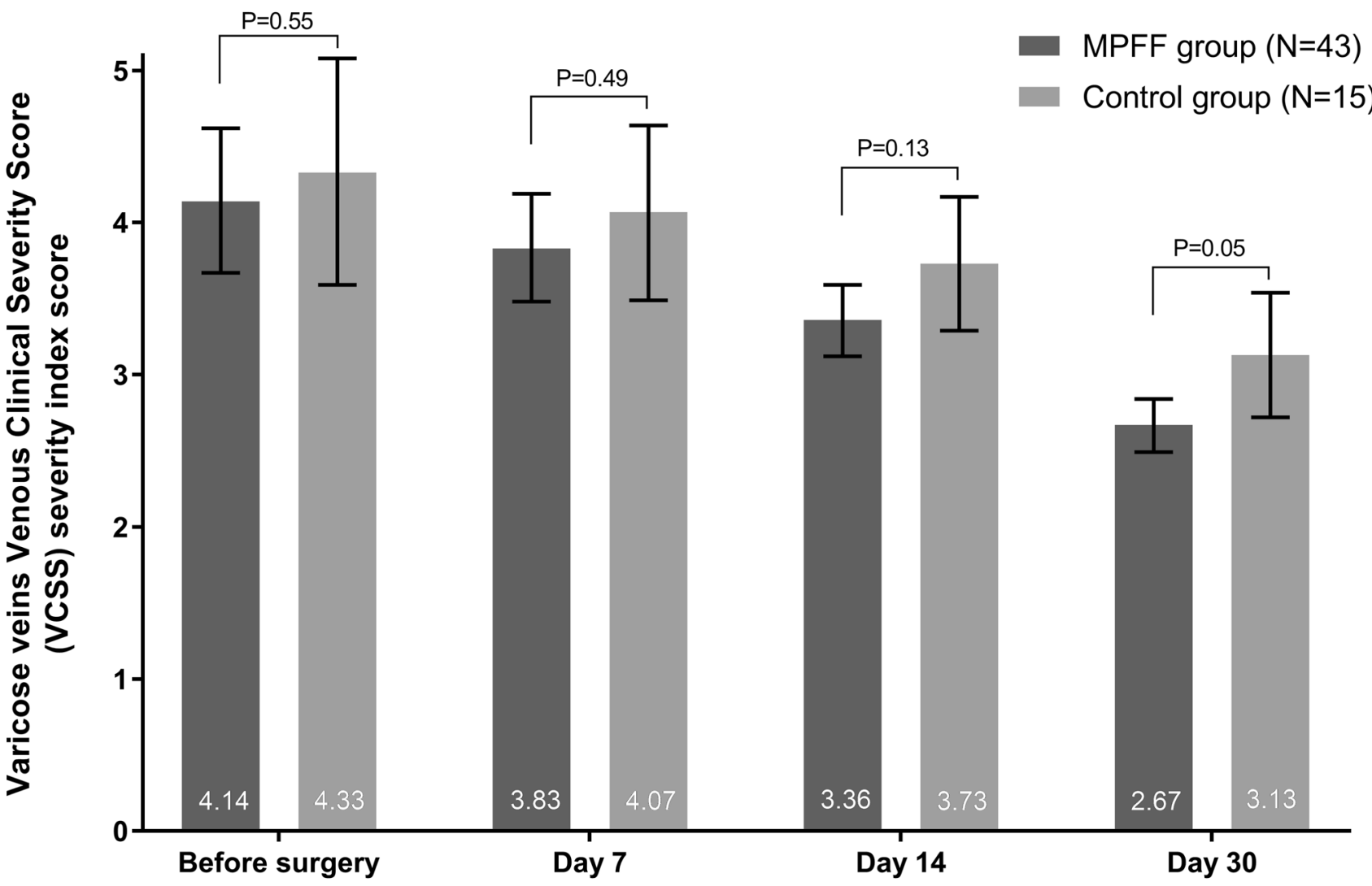

Fig. 2 Varicose veins Venous Clinical Severity Score (VCSS) severity index: comparative cross-group analysis. Error bars represent $95 \%$ confidence intervals. MPFF micronized purified flavonoid fraction

percentage of women in Group A $(58 \%)$ was almost the same as in Group B (60\%). Therefore, this confounder would not have had a major impact on the final result of the evaluation of the MPFF treatment effectiveness in our study.

Compared with other published reports [20-22], our results indicated that MPFF may have a better performance profile in terms of VAS/VCSS scores and the CIVIQ-20 questionnaire; this is probably because of a more sparing non-thermal non-tumescent technology employed to eliminate vertical venous reflux. As it was established, the pain syndrome intensity during edema/swelling is significantly higher than during the surgery itself [30]. Our patients had better perceptions of endovenous intervention, something that we attributed to the fact that MOCA was performed without tumescent anesthesia, only requiring local anesthesia at the single GSV/SSV puncture site, thereby reducing the risk of heat-related nerve injury.

One of the key components of MOCA is the process of venous wall damage by a sclerosing agent to form a dense clot (a 'sclerothrombus'); therefore, the use of adjuvant venoactive therapy after the endovenous intervention is appropriate. Endothelial destruction is accompanied by the expression of pro-inflammatory cytokines and growth factors inducing a local venospecific inflammatory response. Administration of MPFF 2 weeks prior to and for 2 months after sclerotherapy has been shown to lower the levels of inflammation and endothelial dysfunction markers (histamine, C-reactive protein, interleukin-1, tumor necrosis factor- $\alpha$, and endothelial vascular growth factor) in venous blood samples [23].

While our results provide a clear indication of the usefulness of MPFF after MOCA, there are limitations of our study design that restrict the generalizability of our results. These include the open-label nature, the absence of randomization or a placebo control, the moderate sample size of patients, a relatively short follow-up period, and the use of subjective endpoint parameters. The patient decision to enter the study is a potential source of bias. Other outcomes such as the assessment of adherence and a more detailed account of adverse effects would also enhance the study. In addition, MOCA was technically and anatomically successful in $100 \%$ of the patients in this study, thus our study does not provide an opportunity to investigate the impact of MPFF on symptoms and QoL in patients whose MOCA procedure was not successful. Future research should investigate this issue, as well as the potential impact of MPFF 


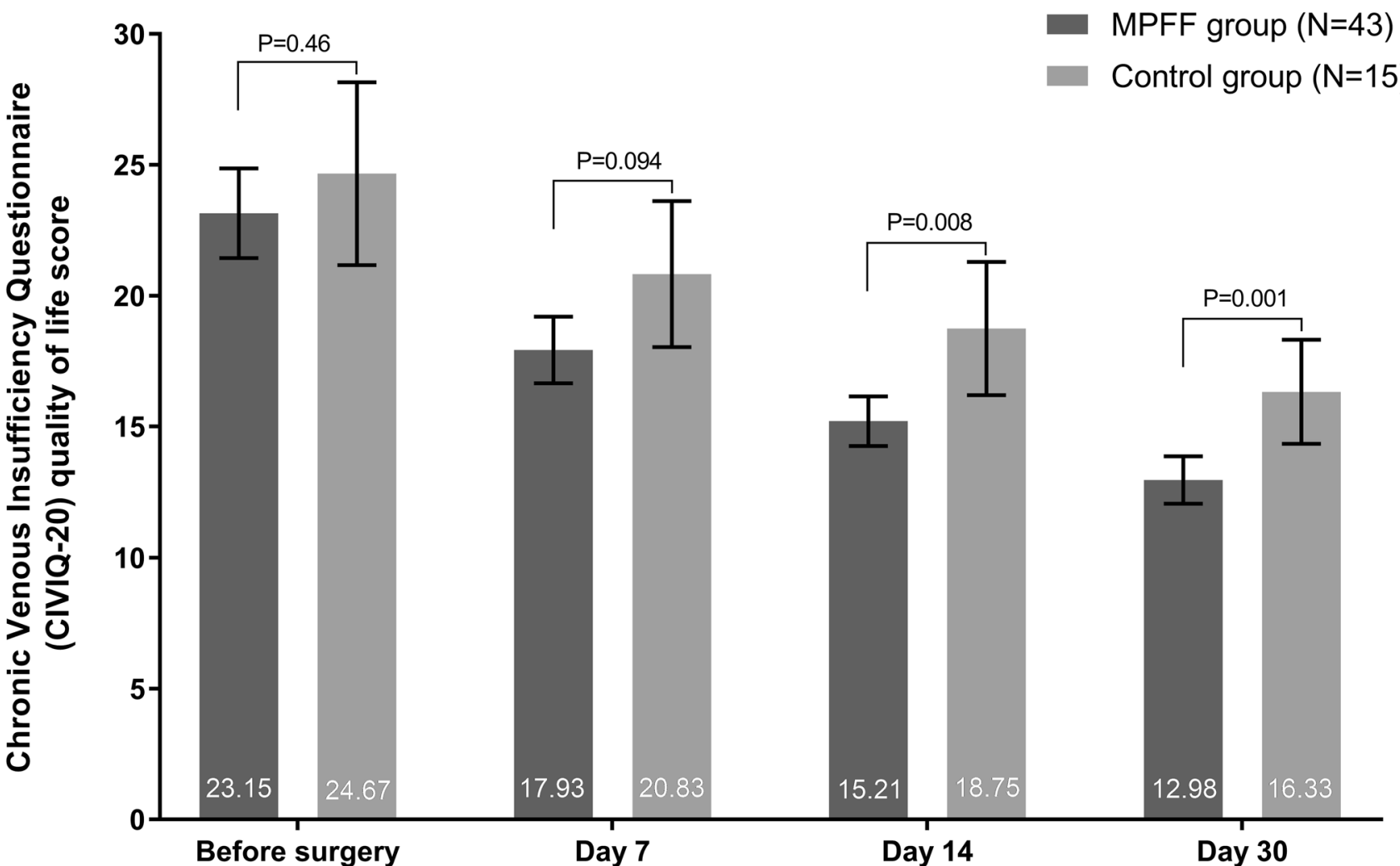

Fig. 3 Chronic Venous Insufficiency Questionnaire (CIVIQ-20) quality-of-life scores: comparative cross-group analysis. Error bars represent 95\% confidence intervals. MPFF micronized purified flavonoid fraction

on post-MOCA occlusion rates, although our data of $100 \%$ anatomical success would suggest that MPFF has no impact on the rate of procedural success.

Strengths of the study were the real-world scenario in which it was conducted. Furthermore, confounding factors were partly mitigated by the fact that in both groups the percent of women was equal to $\sim 60 \%$, the average age was also almost the same, most of the patients in both groups underwent the same surgical operation (MOCA plus mini-phlebectomy). Thus, potential confounders, such as sex, age, and the exact type of surgical operation, have been kept to the minimum.

\section{Conclusions}

Venoactive adjuvant therapy with MPFF at a standard daily dosage of $1000 \mathrm{mg}$ for 4 weeks after endovenous MOCA can significantly alleviate the post-procedural pain syndrome, reduce the severity of the clinical manifestations, and improve QoL. There is a pressing need for prospective, randomized, placebo-controlled trials to assess the subjective and objective efficacy of MPFF after endovenous MOCA.

Supplementary Information The online version contains supplementary material available at https://doi.org/10.1007/s40801-021-00249-4.

Acknowledgements The authors thank Matt Weitz of Springer Healthcare Communications for language and formatting assistance in the preparation of this article. This assistance was funded by Servier France.

\section{Declarations}

Funding The authors received no financial support from pharmaceutical companies or medical device manufacturers for the conduct of this study. Medical writing assistance and open-access publication costs were funded by Servier.

Conflict of interest Vladimir Y. Khryshchanovich, Yuri S. Nebylitsin, and Vladimir A. Kosinets have no conflicts of interest that are directly relevant to the content of this article.

Availability of data and material All data generated or analyzed during this study are included in this published article (and its supplementary information files). 
Ethics approval The study was approved by the Belarusian State Medical University's Biomedical Ethics Committee (approval number 20140455).

Consent to participate Informed consent was obtained from all individual participants included in the study.

Consent for publication Not applicable.

Code availability Not applicable.

Author contributions All authors made substantial contributions to the conception and design of the article, acquisition of data, or analysis and interpretation of data; took part in drafting the article or revising it critically for important intellectual content; gave final approval of the version to be published; and agree to be accountable for all aspects of the work. The corresponding author had full access to the data and final responsibility for the decision to submit for publication.

Open Access This article is licensed under a Creative Commons Attribution-NonCommercial 4.0 International License, which permits any non-commercial use, sharing, adaptation, distribution and reproduction in any medium or format, as long as you give appropriate credit to the original author(s) and the source, provide a link to the Creative Commons licence, and indicate if changes were made. The images or other third party material in this article are included in the article's Creative Commons licence, unless indicated otherwise in a credit line to the material. If material is not included in the article's Creative Commons licence and your intended use is not permitted by statutory regulation or exceeds the permitted use, you will need to obtain permission directly from the copyright holder. To view a copy of this licence, visit http://creativecommons.org/licenses/by-nc/4.0/.

\section{References}

1. Nicolaides A, Kakkos S, Baekgaard N, Comerota A, de Maeseneer $\mathrm{M}$, Eklof B, et al. Management of chronic venous disorders of the lower limbs: guidelines according to scientific evidence. Part II. Int Angiols. 2020;39(3):175-240. https://doi.org/10.23736/ S0392-9590.20.04388-6.

2. Hassanin A, Aherne TM, Greene G, Boyle E, Egan B, Tierney S, et al. A systematic review and meta-analysis of comparative studies comparing nonthermal versus thermal endovenous ablation in superficial venous incompetence. J Vasc Surg Venous Lymphat Disord. 2019;7(6):902-13.e3. https://doi.org/10.1016/j.jvsv.2019. 06.009 .

3. Baccellieri D, Apruzzi L, Ardita V, Favia N, Saracino C, Carta N, et al. Early results of mechanochemical ablation for small saphenous vein incompetency using 2\% polidocanol. J Vasc Surg Venous Lymphat Disord. 2020. https://doi.org/10.1016/j.jvsv.2020.09. 001 .

4. Mosquera-Rey V, Del Castro Madrazo JA, Angeles MHM, Cordeu RA, Azofra EA, Perez MA. Mechanochemical ablation for great and small saphenous veins insufficiency in patients with type III shunt. Phlebology. 2021;36(2):145-51. https://doi.org/10.1177/ 0268355520951695.

5. Nugroho J, Wardhana A, Ghea C. Mechanical occlusion chemically assisted ablation (MOCA) for saphenous vein insufficiency: a meta-analysis of a randomized trial. Int J Vasc Med. 2020;2020:8758905. https://doi.org/10.1155/2020/8758905.

6. Vahaaho S, Halmesmaki K, Mahmoud O, Alback A, Noronen $\mathrm{K}$, Venermo M. Three-year results of a randomized controlled trial comparing mechanochemical and thermal ablation in the treatment of insufficient great saphenous veins. J Vasc Surg Venous Lymphat Disord. 2020. https://doi.org/10.1016/j.jvsv. 2020.08.007.

7. Holewijn S, van Eekeren R, Vahl A, de Vries J, Reijnen M, MARADONA Study Group. Two-year results of a multicenter randomized controlled trial comparing. Mechanochemical endovenous Ablation to RADiOfrequeNcy Ablation in the treatment of primary great saphenous vein incompetence (MARADONA trial). J Vasc Surg Venous Lymphat Disord. 2019;7(3):364-74. https://doi.org/10.1016/j.jvsv.2018.12.014.

8. Tawfik AM, Sorour WA, El-Laboudy ME. Laser ablation versus mechanochemical ablation in the treatment of primary varicose veins: a randomized clinical trial. J Vasc Surg Venous Lymphat Disord. 2020;8(2):211-5. https://doi.org/10.1016/j.jvsv.2019.10. 025.

9. Nicolaides A, Kakkos S, Eklof B, Perrin M, Nelzen O, Neglen $\mathrm{P}$, et al. Management of chronic venous disorders of the lower limbs: guidelines according to scientific evidence. Int Angiol. 2014;33(2):87-208.

10. Wittens C, Davies AH, Baekgaard N, Broholm R, Cavezzi A, Chastanet $\mathrm{S}$, et al. Editor's choice: management of chronic venous disease: clinical practice guidelines of the European Society for Vascular Surgery (ESVS). Eur J Vasc Endovasc Surg. 2015;49(6):678-737. https://doi.org/10.1016/j.ejvs.2015.02.007.

11. Nicolaides A, Kakkos S, Baekgaard N, Comerota A, de Maeseneer M, Eklof B, et al. Management of chronic venous disorders of the lower limbs: guidelines according to scientific evidence. Part I. Int Angiol. 2018;37(3):181-254. https://doi.org/10.23736/S03929590.18.03999-8.

12. Bogachev VY, Boldin BV, Turkin PY. Administration of micronized purified flavonoid fraction during sclerotherapy of reticular veins and telangiectasias: results of the national, multicenter, observational program VEIN ACT PROLONGED-C1. Adv Ther. 2018;35(7):1001-8. https://doi.org/10.1007/s12325-018-0731-z.

13. Quarto G, Amato B, Giani U, Benassai G, Gallinoro E, Apperti $\mathrm{M}$, et al. Comparison of traditional surgery and laser treatment of incontinent great saphenous vein: results of a meta-analysis. Ann Ital Chir. 2016;87:61-7.

14. Martinez MJ, Bonfill X, Moreno RM, Vargas E, Capella D. Phlebotonics for venous insufficiency. Cochrane Database Syst Rev. 2005;3:CD003229. https://doi.org/10.1002/14651858.CD003229. pub2.

15. Nicolaides AN. The benefits of micronized purified flavonoid fraction (MPFF) throughout the progression of chronic venous disease. Adv Ther. 2020;37(Suppl 1):1-5. https://doi.org/10.1007/ s12325-019-01218-8.

16. Gavrilov SG, Moskalenko YP, Karalkin AV. Effectiveness and safety of micronized purified flavonoid fraction for the treatment of concomitant varicose veins of the pelvis and lower extremities. Curr Med Res Opin. 2019;35(6):1019-26. https://doi.org/10.1080/ 03007995.2018.1552043.

17. Jantet G. Chronic venous insufficiency: worldwide results of the RELIEF study. Reflux assEssment and quaLity of lIfe improvEment with micronized Flavonoids. Angiology. 2002;53(3):245-56. https://doi.org/10.1177/000331970205300301.

18. O'Donnell TF Jr, Passman MA, Marston WA, Ennis WJ, Dalsing M, Kistner RL, et al. Management of venous leg ulcers: clinical practice guidelines of the Society for Vascular Surgery ${ }^{\circledR}$ and the American Venous Forum. J Vasc Surg. 2014;60(2 Suppl):3S-59S. https://doi.org/10.1016/j.jvs.2014.04.049.

19. Mansilha A, Sousa J. Benefits of venoactive drug therapy in surgical or endovenous treatment for varicose veins: a systematic review. Int Angiol. 2019;38(4):291-8. https://doi.org/10.23736/ S0392-9590.19.04216-0.

20. Pokrovsky AV, Saveljev VS, Kirienko AI, Bogachev VY, Zolotukhin IA, Sapelkin SV, et al. Surgical correction of varicose vein 
disease under micronized diosmin protection (results of the Russian multicenter controlled trial DEFANS). Angiol Sosud Khir. 2007;13(2):47-55

21. Stoiko YM, Mazaishvili KV, Khlevtova TV, Tsyplyashchuk AV, Kharitonova SE, Akimov SS. Effect of pharmacotherapy on course of postoperative period after endovenous thermal ablation. Angiol Sosud Khir. 2015;21(3):77-81.

22. Bogachev V, Golovanova OV, Kuzhetsov AN, Shekoian AO. On advisability of perioperative phleboprotection in endovascular treatment of lower in varicose disease: first initial results of the decision study. Angiol Sosud Khir. 2012;18(2):90-5.

23. Bogachev VY, Boldin BV, Lobanov VN. Benefits of micronized purified flavonoid fraction as adjuvant therapy on inflammatory response after sclerotherapy. Int Angiol. 2018;37(1):71-8. https:// doi.org/10.23736/S0392-9590.17.03868-8.

24. Eklof B, Rutherford RB, Bergan JJ, Carpentier PH, Gloviczki $\mathrm{P}$, Kistner RL, et al. Revision of the CEAP classification for chronic venous disorders: consensus statement. J Vasc Surg. 2004;40(6):1248-52. https://doi.org/10.1016/j.jvs.2004.09.027.

25. Tessari L, Cavezzi A, Frullini A. Preliminary experience with a new sclerosing foam in the treatment of varicose veins. Dermatol Surg. 2001;27(1):58-60.

26. Ciostek P, Kowalski M, Woźniak W, Miłek T, Myrcha P, Migda B. Phlebogriffe: a new device for mechanochemical ablation of incompetent saphenous veins: a pilot study. Phlebol Rev. 2015;23(3):72-7. https://doi.org/10.5114/pr.2015.57466.

27. Passman MA, McLafferty RB, Lentz MF, Nagre SB, Iafrati MD, Bohannon WT, et al. Validation of Venous Clinical Severity Score (VCSS) with other venous severity assessment tools from the American Venous Forum, National Venous Screening Program. J Vasc Surg. 2011;54(6 Suppl):2S-9S. https://doi.org/10.1016/j. jvs.2011.05.117.

28. Gloviczki P, Comerota AJ, Dalsing MC, Eklof BG, Gillespie DL, Gloviczki ML, et al. The care of patients with varicose veins and associated chronic venous diseases: clinical practice guidelines of the Society for Vascular Surgery and the American Venous Forum. J Vasc Surg. 2011;53(5 Suppl.):2S-48S. https://doi.org/ 10.1016/j.jvs.2011.01.079.

29. van Eekeren RR, Boersma D, Konijn V, de Vries JP, Reijnen MM. Postoperative pain and early quality of life after radiofrequency ablation and mechanochemical endovenous ablation of incompetent great saphenous veins. J Vasc Surg. 2013;57(2):445-50. https://doi.org/10.1016/j.jvs.2012.07.049.

30. Allaert FA. Meta-analysis of the impact of the principal venoactive drugs agents on malleolar venous edema. Int Angiol. 2012;31(4):310-5. 